\title{
Therapeutic STING activation boosts microglia phagocytosis and ameliorates depression-like behaviors during chronic restraint stress
}

\section{Qian Zhai}

Xi'an Jiaotong University Medical College First Affiliated Hospital

\section{Yanpeng Zhang}

Xi'an Jiaotong University Medical College First Affiliated Hospital

Shuwen Tan

Xi'an Jiaotong University Medical College First Affiliated Hospital

Jianyu Sun

Xi'an Jiaotong University Medical College First Affiliated Hospital

\section{Mao Ye}

Xi'an Jiaotong University Medical College First Affiliated Hospital

Hui Gao

Xi'an Jiaotong University Medical College First Affiliated Hospital

Kairui Pu

Xi'an Jiaotong University Medical College First Affiliated Hospital

\section{Meiyan Wu}

Xi'an Jiaotong University Medical College First Affiliated Hospital

Qiang Wang ( $\square$ dr.wangqiang@139.com )

The First affiliated Hospital of Xi'an Jiaotong University https://orcid.org/0000-0002-3637-2063

\section{Research}

Keywords: microglia, phagocytosis, interferons, neuroinflammation, chronic stress

Posted Date: July 14th, 2021

DOI: https://doi.org/10.21203/rs.3.rs-695179/v1

License: (c) (i) This work is licensed under a Creative Commons Attribution 4.0 International License. Read Full License 


\section{Abstract \\ Background}

The STING-TBK1-IRF3 signaling pathway involves in modulating host innate immunity, however, the potential role of STING signaling pathway in chronic restraint stress model has not been determined. The aim of this study is to explore the underlying role of STING signaling pathway in regulating neuroinflammation, as well as to evaluate the therapeutic potential of STING agonist during chronic restraint stress.

\section{Methods}

C57BL/6 mice were subject to 14-day intermittent restraint stress. Sucrose preference, elevated plus maze and tail suspension tests were measured in chronic restraint stress mice. Expression levels of proinflammatory cytokines were tested by QT-PCR and Luminex cytokine assays. The fluorescencelabeled latex beads, flow cytometry and CD68 positive cell counts were utilized to evaluate phagocytic abilities of microglia. Then, the ability of intracerebroventricular injection of STING agonist, 2'3-cGAMP, to reverse the depression-like behaviors and inflammatory cytokines was examined.

\section{Results}

We found that the expression levels of STING, p-TBK1, and p-IRF3 were remarkably decreased in chronic restraint stress mice, which was associated with decreased IFN- $\beta$ secretion. Moreover, the STING agonist, 2'3-cGAMP, significantly alleviated the neuroinflammation and ameliorated depression-like behavior which depends on the functional STING activation. Furthermore, 2'3-cGAMP promoted microglia phagocytosis through cGAMP-STING-dependent IFN- $\beta$ release, which was essential for recovery from neuroinflammation during chronic restraint stress.

\section{Conclusions}

These findings demonstrate that STING signaling pathway is a critical mediator in regulating microglia phagocytosis and may serve as a novel therapeutic target for chronic stress-related psychiatric diseases.

\section{Background}

Psychosocial stress plays an important role in the pathophysiology of stress-related psychiatric diseases such as depression and anxiety disorders[1], and leads to various health problems including immune deficiencies, digestive disorders and cardiovascular diseases[2, 3]. Chronic restraint stress causes activation of the brain's immune responses manifested in microglia activity and leads to depression-like behavior. During this process, endogenous molecules released from neurons upon stress could result in 
profound microglia alterations characterized by number, morphology and function[4, 5]. Several innate immune receptors of microglia have previously been implicated in the chronic stress-induced immune response. For instance, a recent study has suggested crucial roles of TLR2/4 in medial prefrontal cortex for repeated social defeat stress-induced social avoidance[6]. And activation of P2X7 receptor and the NLRP3 inflammasome in hippocampus mediates depression-like behavior induced by chronic stress[7]. A deeper understanding of the roles of intracellular signaling pathways of microglia in chronic stress is useful to optimize preventive and therapeutic strategies to combat the increasing incidence of psychosocial diseases.

Stimulator of interferon gene (STING), as an adaptor protein, predominantly resides in the endoplasmic reticulum and regulates innate immune signaling processes by detecting aberrant cytosolic DNA[8, 9]. Previous studies have reported that chronic stress triggers certain biological pathways that ultimately result in the accumulation of DNA damage[10], and a cytosolic DNA sensing pathway (especially cGASSTING pathway) is the major link between DNA damage and innate immunity[11, 12]. STING subsequently initiates further signaling cascades by recruiting and activating TANK-binding kinase 1 (TBK1) and transcription factor interferon regulatory factor 3 (IRF3), ultimately leading to the production of interferon- $\beta$ (IFN- $\beta)[13,14]$. Intriguingly, STING was exclusively expressed by microglia in the brain[15]. STING signaling pathway has been implicated in neuroinflammation during neurodegeneration, but its mechanism of induction and its consequences in chronic restraint stress model remain unclear.

In the current study, we found that chronic restraint stress induces a transient proliferation and activation of microglia, accompanied with the increase of inflammatory cytokines in brain. Moreover, STING is downregulated in chronic restraint stress mice and STING agonist cGAMP induces a type I interferon response in microglia which depends on a functional STING pathway. Treatment with cGAMP enhances microglia phagocytosis by inducing the production of IFN- $\beta$, relieves neuroinflammation and ameliorates depression-like behaviors during chronic restraint stress. Collectively, our findings identify the STING pathway as a regulator of microglia phagocytosis and uncover a potential use of STING agonists for the treatment of stress-related mood disorders.

\section{Methods}

\section{Animals}

\section{RST protocol}

The Experimental Animal Ethics Committee of Xi'an Jiaotong University has approved all the animal experiments involved in this study. Eight-week-old male wild-type C57BL/6 mice weighing between 20-23 $\mathrm{g}$ were housed in a specific pathogen-free animal faculty of the Center for Brain Science, the First Affiliated Hospital of Xi'an Jiaotong University. According to an established paradigm, the restraint stress experiments were performed after 1 week of habituation. The RST mice were placed in well-ventilated 
plastic tubes to restrict the movement of the limbs for 6 hours (from 9:30 AM to 15:30 PM) per day lasting 14 days.

\section{Behavioral tests}

All behavioral tests were performed during the afternoon in a dedicated sound-proof behavioral facility by researchers blind to treatment. Mice were brought to the procedure room $1 \mathrm{~h}$ before the start of each behavioral test and remained in the same room throughout the test. At all times, sound was masked with 55-60 Db of white noise. Mice were allowed to rest for one day after the completion of the 14-day RST protocol.

Sucrose preference tests were performed on day 16. A bottle with tap water and another bottle with $2 \%$ sucrose solution were given to mice. The mice had been trained (given a free choice between two bottles) every day to avoid neophobia. To prevent a possible effect of behavior, the left/right location of the bottles was switched every day. Preference was calculated by determining the percentage of sucrose consumed divided by the total fluid intake (sucrose intake/total fluid intake $\times 100 \%$ ).

Tail suspension tests were performed on day 18. As previously described[16],mice were suspended individually by their tails from a metal rod fixed $30 \mathrm{~cm}$ above the surface of a table. The tip of the tail was fixed using adhesive tape (the distance from tip of the tail was $2 \mathrm{~cm}$ ). The subject mouse was suspended by the tail for $6 \mathrm{~min}$. Mice were acclimated for the first $1 \mathrm{~min}$, and immobility time was measured for the last $5 \mathrm{~min}$. Immobility was defined as no movement of the limbs and tails. Mice that climbed up their tails were excluded from the experiment.

An elevated plus maze test was performed on a plus-shaped apparatus with two open and two closed arms. The maze was elevated $50 \mathrm{~cm}$ from the floor. Mice were tested in a single 5-min session. The time stayed in open arms and the number of entries into the open arms was scored using a video-tracking system (Smart 3.0). Between each trial, the maze was cleaned with 75\% ethanol.

\section{Microglial cell density and morphological analyses}

Pictures were taken throughout the entire $12 \mu \mathrm{m}$ thickness of the slice by Z-stack, covering the whole surface of the region (prefrontal cortex and hippocampus). All images from a given slice were acquired with the same exposure time and acquisition parameters. Unbiased stereological counting of Iba1positive cells was done using a modified version of the optical fractionator as was previously described[5]. The prefrontal cortex and hippocampus borders were delineated under low magnification and the stereo-investigator 10 software (MBF Bioscience) was used to determine the surface area and to count cells. The software automatically calculates the number of Iba1-labeled cells within the region of interest.

Microglial morphologic analysis was performed in the blind using ImageJ software (NIH, Bethesda, Maryland, USA). Briefly, confocal images for the selected marker lba-1 were modified as 8-bit projection images. The resulting images were smooth processed, binarized and skeletonized, using the Skeletonize 
Plugin for ImageJ[17]. For each microglia cell, a polygonal region of interest (ROI) that circumscribed all microglial processes belonging to that cell was set. From each ROI a single cell image was generated, single cell image was subjected to Skeletonize Plugin. To determine the 'number of branches', the 'number of junctions', and 'the maximum branch length' the particle-filtered image was processed by choosing the Analyze Skeleton 2D/3D option in the Skeletonize Plugin, and analyzed using the Fiji plugin 'Analyze Skeleton' to obtained the data from the results table.

\section{Microglia isolation and flow cytometry}

Brains were dissected into $1-2 \mathrm{~mm}^{3}$ pieces with small scissors and incubated for $30 \mathrm{~min}$ at $37^{\circ} \mathrm{C}$ in $1 \mathrm{ml}$ HBSS solution containing 2\% FBS, $1 \mathrm{mg} / \mathrm{ml}$ Collagenase D and $50 \mu \mathrm{g} / \mathrm{ml}$ DNase $\mathrm{l} .10 \mathrm{mM}$ EDTA was added to the $1.5 \mathrm{~mL}$ tube to stop the enzymatic reaction and homogenates were pipetted for further dissociation. Next the homogenates were filtered through a nylon mesh (70 $\mu \mathrm{m}$ pore size) in a $50 \mathrm{~mL}$ tube using the plunger of a $10 \mathrm{~mL}$ syringe, washed with cold FACS buffer ( $2 \%$ FBS, 1 mM EDTA in PBS without $\mathrm{Ca}^{2+}$ or $\mathrm{Mg}^{2+}$ ) and centrifuged at $300 \mathrm{~g}$ at room temperature for $5 \mathrm{~min}$. For the enrichment of microglia, the cell pellet was resuspended with $30 \%-37 \%-70 \%$ Percoll density gradient medium and centrifuged at $800 \mathrm{~g}$, no acceleration and braking, at room temperature for $40 \mathrm{~min}$. Next, the $70-37 \%$ density gradient interphase containing the macrophage subpopulations cells was collected into a clean tube, washed with $5 \mathrm{ml} \mathrm{FACS}$ buffer and centrifuged at $500 \mathrm{~g}$ at $4^{\circ} \mathrm{C}$ for $5 \mathrm{~min}$, followed by antibody labeling and flow cytometry analysis. Antibodies against PerCP-CD11b (Biolegend, San Diego, CA), PE-CD45 (Biolegend, San Diego, CA), CD16/32 (Biolegend, San Diego, CA), fixable viability dye eFluor 520 (ebioscience, San Diego, CA) were used. Analysis was performed on a Calibur (BD Biosciences, BD Diva Software) and analyzed with FlowJo software.

\section{Intracerebroventricular injection of 2'3-cGAMP}

Animals were anesthetized with $4 \%$ chloral hydrate for a surgical plane of anesthesia throughout the procedure. Animals were placed in a stereotaxic apparatus (RWD Life Science, Shenzhen, China) with the ear bars in the ear canals and the incisors in the tooth bar of the mouse adapter. After making an approximately $1-\mathrm{cm}$ incision in the scalp, the periosteum was scrubbed from the skull with sterile cottontipped applicators to reveal the bregma. Hamilton syringes fitted with 22-gauge Huber point removable needles were filled with $10 \mu \mathrm{l}$ of saline with or without 2'3-cGAMP (diluted from $20 \mu \mathrm{g} / \mathrm{ml}$ in saline, Abcam, Cambridge, UK). The needle was positioned over the bregma and then moved to coordinates 0.22 $\mathrm{mm}$ anterior, $1.0 \mathrm{~mm}$ right, and $2.5 \mathrm{~mm}$ down after the bevel of the needle disappeared through the skull. The needle was injected until the resistance decreased slightly, indicating that the needle had penetrated into the lateral ventricle. 2'3-cGAMP $(2.5 \mu \mathrm{l}, 20 \mu \mathrm{g} / \mathrm{ml})$ was injected gradually by hand over approximately $8 \mathrm{~min}$. After $5 \mathrm{~min}$, the needle was backed out of the skull while applying downward pressure on the animal's skull with a sterile cotton-tipped applicator. The incision was sutured with 1 horizontal mattress stitch using $6-0$ suture. Animals were allowed to recover from the anesthesia in their home cage. After 5 days, mice were placed in well-ventilated plastic tubes to restrict the movement of the limbs for 6 hours per day lasting 14 days.

\section{BV2 microglia cell culture and treatment}


Mouse BV2 microglia cells were purchased from Procell Culture Collection (Wuhan, China). The cells were cultured in Dulbecco's modified eagle's medium (DMEM, Gibco, USA) containing 10\% heat-inactivated FBS (Gibco, USA) and 100 units $/ \mathrm{ml}$ penicillin and $0.1 \mathrm{mg} / \mathrm{mL}$ streptomycin in a humidified condition of $5 \% \mathrm{CO} 2$ and $95 \%$ air at $37^{\circ} \mathrm{C}$. The medium was changed every 3 days. Cells were grown to $80 \%$ confluence in dishes for experiments involving insult with various cytokines, including lipopolysaccharide (LPS, 1 ug/ml, Sigma), 2'3-cGAMP (200 ng/ml, Abcam), recombinant IFN- $\beta$ (100ng/ml, R\&D Systems), recombinant murine interferon-y (10 ng/ml, Peprotech), interleukin-10 (10 ng/ml, Peprotech), interleukin-4 (10 ng/ml, Peprotech), and interleukin-13 (10 ng/ml, Peprotech).

\section{Assessment of phagocytosis}

Phagocytosis was assessed in BV2 microglia cell line using amino-modified polystyrene latex beads (1.0$\mu \mathrm{m}$ mean particle size, Sigma-Aldrich, Boston, MA) as the phagocytosis target. Microglia were plated at the concentration of $1 \times 10^{4}$ cells in a confocal dish and treated with 2'3-cGAMP $(200 \mathrm{ng} / \mathrm{ml}$ in the culture medium) for $24 \mathrm{~h}$. Then $0.5 \mu \mathrm{L}$ of fluorescent latex beads were re-suspended in medium for $2 \mathrm{~h}$ at $37^{\circ} \mathrm{C}$. After $2 \mathrm{~h}$ of incubation, $1 \mathrm{~mL}$ of cold PBS was added and washed three times until non-phagocytosed beads were removed. Bead intake was quantified by measuring the raw intensity density using the Fiji software and maintaining the same threshold restrictions for all the experimental conditions. We characterized microglia phagocytosis as the amount of internalized bioparticles per cell in actively engulfing cells. In all cases, quantifications were performed in 50 cells from 5 fields per experimental condition per experiment, with five independent experiments being performed.

Phagocytosis of fluorogenic E. coli particles (pHrodo Green, Invitrogen) was analyzed using BV2 microglial cells. Briefly, cells were plated in 6-well plates at a density of $2 \times 10^{6}$ cells and cultured for 24 to 48 hours. pHrodo E. coli bioparticles were dissolved in PBS to a concentration of $1 \mathrm{mg} / \mathrm{ml}$, and a total of $100 \mu \mathrm{g}$ of bioparticles was added per well and incubated for $60 \mathrm{~min}$ at $37^{\circ} \mathrm{C}$. As a negative control, phagocytosis was inhibited with $10 \mathrm{mM}$ cytochalasin $\mathrm{D}$, which was added 30 min before addition of pHrodo E. coli bioparticles. Cells were harvested by trypsinization, washed two times with FACS sample buffer, and analyzed by flow cytometry on a BD FACS Canto ${ }^{\text {TM }}$ II flow cytometer. Data analysis was performed using the FlowJo software.

\section{siRNA knockdown of STING or IFN $\beta$}

Custom double-stranded STING or IFN $\beta$ siRNA primers were purchased from GenePharma (shanghai, China). Three primer pairs were ordered to maximize the probability of achieving successful STING or IFN $\beta$ knockdown. See Supplemental Materials and Methods, which presents the necessary methods used in this study.

\section{Luminex cytokine assays}

See Supplemental Materials and Methods, which presents the necessary methods used in this study.

\section{Western blot analysis and Immunofluorescence}


See Supplemental Materials and Methods, which presents the necessary methods used in this study.

\section{Quantitative real-time PCR}

Hieff® qPCR SYBR® Green Master Mix was used for quantitative PCR. See Supplemental Materials and Methods, which presents the necessary methods used in this study.

\section{Statistical analysis}

All statistical analyses were performed using GraphPad Prism 6.0. In all cases, data are presented as the mean \pm SEM. Two-tailed unpaired independent t-test was performed to compare two groups. One-way ANOVA was performed to evaluate the significance differences between multiple groups, and Bonferroni post hoc tests were performed following significant main effects or interactions in ANOVA where applicable. Two-way ANOVA was performed to evaluate the significant differences with conditions (CON vs RST), treatments (vehicle vs cGAMP) or an interaction (stress by cGAMP). At least three independent repeated experiments are performed. ${ }^{\star} \mathrm{P}<0.05 ; * \star \mathrm{P}<0.01 ; * \star \star \mathrm{P}<0.001$.

\section{Results}

\section{Chronic restraint stress induces depression-like behaviors and neuroinflammation}

During the 14-day experimental period with 6-h restraint stress daily (Fig. 1a), the body weight of RST mice was significantly decreased (from $22.5 \pm 0.31 \mathrm{~g}$ to $20.4 \pm 0.46 \mathrm{~g}$ ) in the first 5 days compared to CON mice (from $22.4 \pm 0.32 \mathrm{~g}$ to $23.2 \pm 0.45 \mathrm{~g}$, Fig. 1b). The total food intake of RST mice was reduced markedly during the first three days (from $16.0 \mathrm{~g}$ to $12.2 \mathrm{~g}$ ). However, food intake of RST mice was almost equal with CON mice by day 4, since the food intake of RST mice showed a tendency to increase from the 5th to the 14th day. Next, we observed the behavioral consequences in CON and RST mice after they completed the 14-day paradigm. RST mice displayed anhedonia in a sucrose preference test compared to CON mice $(P<0.01$, Fig. 1c). Moreover, the tail suspension test revealed a significant increase of the immobility time in RST mice (CON vs. RST, $126.28 \pm 10.60$ s vs. $160.60 \pm 8.68$ s, P<0.05, Fig. 1d). In the elevated plus maze test, RST mice spent relatively less time in the open arms (CON vs. RST, $71.26 \pm 10.47$ s vs. $40.05 \pm 6.72$ s, $P<0.05$, Fig. 1e). Meanwhile, the total number of open arm entries was also decreased in RST mice $(P<0.05$, Fig. 1e). Taken together, these data indicated that chronic restraint stress results in depression-like behaviors.

To further assess whether chronic restraint stress induces neuroinflammation in mice, we measured the mRNA expression of inflammatory cytokines in the prefrontal cortex and hippocampus. We found that the mRNA expression of TNF-a was increased to 2.12 \pm 0.12 -fold in the prefrontal cortex tissue of RST mice compared to that in CON mice $(P<0.001)$. Similarly, IL-1 $\beta$ expression was increased to $1.78 \pm 0.26$ fold and IL-6 expression was increased to $1.56 \pm 0.21$-fold in the RST group $(P<0.05)$. In addition, CXCL10 and CCL2 expression levels were also significantly increased in RST mice (Fig. 1f). Similarly, the 
mRNA expression of inflammatory cytokines in the hippocampus of RST mice were also increased. These results revealed that chronic restraint stress can enhance the release of pro-inflammatory cytokines in prefrontal cortex and hippocampus.

\section{Microglia are activated towards pro-inflammatory phenotype during chronic restraint stress}

To consider the effect of chronic restraint stress on microglia density and morphology, we then analyzed changes in microglia cell number by counting Iba1 stained cells and morphology by counting branches and junctions per cell. Our data indicated that the lba1-positive cell number was significantly higher in the prefrontal cortex (CON vs. RST, $7743 \pm 159$ vs. $9873 \pm 141$ cells $/ \mathrm{mm}^{3}, \mathrm{P}<0.01$ ) and hippocampus (CON vs. RST, $6228 \pm 139$ vs. $7531 \pm 130$ cells $/ \mathrm{mm}^{3}, \mathrm{P}<0.05$ ) of RST mice (Fig. S1a-c). The analysis of cell morphology in the prefrontal cortex and hippocampus of RST mice revealed a more ramified phenotype, with a higher number of branches and junctions per cell $(P<0.001$, Fig. S1d,e). However, there was no change in the maximum branch length of microglia between RST mice and CON mice (Fig. S1f). Consistently, flow cytometry analysis revealed $\mathrm{Cd} 11 \mathrm{~b}^{\text {high }} \mathrm{CD} 45^{\text {low }}$ microglia number was increased in RST mice compared to CON mice $(P<0.05$, Fig. S1g). As we known, the M1 phenotype is more likely associated with uncontrolled neuroinflammation, while the M2 phenotype promotes inflammation resolution and tissue repair. Interestingly, we found that the expression levels of M1 microglia markers induced nitric-oxide synthase (iNOS, $\mathrm{P}<0.01$ in prefrontal cortex, $\mathrm{P}<0.05$ in hippocampus) and interleukin-6 (IL-6, $\mathrm{P}<0.05$ in prefrontal cortex, $\mathrm{P}<0.01$ in hippocampus) were significantly increased in RST mice (Fig. 2a-c). In contrast, M2 microglia markers brain-derived neurotrophic factor (BDNF, $\mathrm{P}<0.001$ in prefrontal cortex, $\mathrm{P}<0.001$ in hippocampus) and arginase-1 (Arg-1, $\mathrm{P}<0.05$ in prefrontal cortex, $\mathrm{P}<$ 0.05 in hippocampus) expressions were reduced in RST mice (Fig. 2d,e). Above all, these results suggested that microglia are increased in number and more activated towards M1 phenotype during chronic restraint stress.

STING and its downstream effectors p-TBK1 and p-IRF3 protein expressions are inhibited in chronic restraint stress mice

To verify whether STING was critically associated with the change of microglia phenotypes, we used lipopolysaccharide and interferon- $\gamma$ as $M 1$ triggers and interleukin-4 and interleukin-13 as $M 2$ triggers to stimulate BV2 microglia. We observed that STING protein expression was increased by IL-4/IL-13 treatment $(1.7 \pm 0.2$-fold, control vs. IL-4/IL-13) and inhibited by LPS/IFN- $\gamma$ treatment $(0.4 \pm 0.1$-fold, control vs. LPS/IFN-y) (Fig. 2f,g). To further demonstrate the role of the STING signaling pathway in vivo, we measured STING, p-TBK1, and p-IRF3 protein expressions in the prefrontal cortex and hippocampus of RST mice. The immunofluorescence staining assay first revealed that the expression of STING was significantly decreased in the prefrontal cortex and hippocampus of RST mice (Fig. S2a,b). This observation was further confirmed by western blotting for STING protein expression after chronic restraint stress. The result showed that the protein expression level of STING was very low in the prefrontal cortex $(P<0.001)$ and hippocampus $(P<0.01)$ of RST mice (Fig. S2c,d). Moreover, the phosphoprotein TBK1 ( $p-$ 
TBK1) and the phosphoprotein IRF3 (p-IRF3) protein levels were also decreased in the prefrontal cortex and hippocampus of RST mice Fig. S2e,f). Hence, these results suggested that a dominant proinflammatory microglia phenotype is might characterized by a suppression of the STING signaling pathway during chronic restraint stress.

Treatment with cGAMP ameliorates depression-like behaviors and inhibits production of proinflammatory cytokines through the STING-TBK1-IRF3 axis

We next injected the STING agonist 2'3'-cGAMP into the lateral ventricles and assessed the extent of neuroinflammation and depression-like behaviors in mice. As shown in Fig. 3a and 3b, the expression of STING was markedly inhibited in the prefrontal cortex and the hippocampus of RST mice treated with saline, while 2'3'-cGAMP administration partly rescued the expression of STING in RST mice. Consistently, western blotting assays confirmed that the expression of STING was increased in the CGAMP + RST group compared with that in the vehicle + RST group $(P<0.01$ in the prefrontal cortex and $P<0.01$ in the hippocampus, respectively, Fig. 3c,d), while STING expression showed no difference between the cGAMP group and the cGAMP + RST group. Likewise, cGAMP administration directly activates STING resulted in similar phosphorylation of TBK1 and IRF3 in RST mice (Fig. 3e,f). We also performed RT-PCR to examine the mRNA levels of inflammatory cytokines in the brain including TNF-a, IL-1 $\beta, I L-6, I L-10, C C L 2$ and CXCL10. Pro-inflammatory cytokines TNF- $\alpha$, IL-1 $\beta$ and IL- 6 were higher in vehicle + RST mice, and this effect was nearly abolished in cGAMP + RST mice (Fig. 4a,b). Unexpectedly, analysis of serum inflammatory cytokine levels was consistent with changes in the brain (Fig. S3). Our data showed that STING agonist 2'3'-cGAMP induced less inflammatory cytokines production in RST mice. Importantly, RST mice treated with 2'3'-cGAMP displayed more sucrose consumption in the sucrose preference test, lower immobility in tail suspension test and more times in open arms in the elevated plus maze than vehicle + RST mice (Fig. 4c-e). Collectively, these results suggested that STING activation significantly alleviates neuroinflammation and ameliorates restraint stress induced-depression-like behaviors through the STING-TBK1-IRF3 axis.

\section{Microglia phagocytosis is enhanced through cGAMP- STING-dependent IFN- $\beta$ release during chronic restraint stress}

Previous study demonstrated that CGAMP administration enhanced the expressions of type IIFN and IFNrelated genes[18]. IFN- $\beta$ produced by microglia activates phagocytic activity and increases the clearance of myelin debris, which is suggested to cause downregulation of pro-inflammatory cytokines. To determine whether STING signaling pathway was involved in microglia phagocytosis and whether STING activation could affect the phagocytic activity by inducing the release of IFN- $\beta$, we assessed microglia phagocytosis and measured the production of IFN- $\beta$ in vitro and in vivo. In vitro, STING was successfully knocked down by using siRNA transfection method (Fig. 5a). Phagocytosis of fluorescent beads analyses indicated that LPS-stimulated microglia treated with cGAMP displayed an increase red-bead occupancy 
compared with LPS-stimulated microglia (LPS vs. LPS + cGAMP $19.8 \pm 6.3 \%$ vs. $32 \pm 7.9 \%, P<0.01$ ), while a decrease red-bead occupancy was observed in LPS + cGAMP + siRNA group (LPS + cGAMP vs. LPS + cGAMP + siRNA, $19.8 \pm 6.3 \%$ vs. $13.7 \pm 3.04 \%, P<0.001$, Fig. $5 b, c)$. The cellular IFN- $\beta$ production of externally added cGAMP are expected to be similarly affected. We observed that the levels of IFN- $\beta$ were significantly increased in LPS + CGAMP group compared with that in LPS group $(P<0.05$, Fig. $5 d)$. In vivo, we then assessed the number of CD68-positive phagocytic microglia and measured the IFN- $\beta$ expression in the brain. Our results indicated that CD68-positive phagocytic microglia number were significantly increased in the CGAMP + RST group, although the CD68-positive microglia numbers were not changed in the cGAMP group compared with the vehicle group (Fig. 5e-h). The IFN- $\beta$ mRNA expression in the prefrontal cortex was significantly increased in cGAMP-treated RST mice compared with vehicle + RST mice $(P<0.001$, Fig. 5i).

To further clarify whether the phagocytic machinery was dependent on the cGAMP-STING-IFN- $\beta$ signaling, microglia were treated with recombinant IFN $\beta$ (r-IFN $\beta$ ) or STING agonist CGAMP and incubated with pHrodo-Green labeled E. coli bioparticles for measurement of phagocytosis efficiency by flow cytometry. We found that LPS-stimulated microglia phagocytosed E. coli bioparticles more efficiently following treatment with r-IFN $\beta$ or STING agonist cGAMP $(P<0.05$, Fig. $5 \mathrm{j})$. In contrast, treatment with cGAMP, the IFNß-knockdown microglia exhibited a decrease in phagocytotic rates.

In order to better understand the interaction between microglia phagocytosis and depression-like behavior when STING pathway was activated, a Pearson correlation analysis was conducted to examine whether there is relationship between CD68 positive cell numbers, TNF-a or IL-6 level and times in open or closed in the elevated plus maze test (Fig. 6). In the cGAMP-treated restraint stress mice, we observed significant negative correlation between phagocytic microglia cell counts and decreased plasma TNF-a level $(r=-$ $0.599, P=0.011$, Fig. 6a), decreased plasma IL-6 level $(r=-0.597, P=0.015$, Fig. 6b). Moreover, $a$ significant strong positive correlation was observed between phagocytic microglia cell counts and times in open arms of depressive-like behavior $(r=0.534, P=0.027$, Fig. $6 c)$. However, there was no significant correlation between phagocytic microglia cell counts and times in closed arms $(r=0.116, P=0.656$, Fig. $6 \mathrm{~d}$ ). Taken together, these data indicated that cGAMP-STING-dependent IFN- $\beta$ signaling is required to stimulate microglia phagocytosis, and STING activation might alleviate chronic restraint stress-induced neuroinflammation and depression-like behavior through boosting microglia phagocytosis.

\section{Discussion}

Chronic restraint stress produced significant physiological and behavioral changes, notably reduced weight gain and depression-like behaviors. These changes were also accompanied by signs of microglia activation in the brain. This is consistent with past evidence that exposure to stress can trigger a neuroinflammatory response[3]. To our knowledge, neuroinflammation heavily relies on innate immune responses that are primarily mediated by microglia. As a signaling hub in innate immunity, STING is a key adaptor molecule in orchestrating the body's response to inflammation, cell death and autophagy[19, 20]. Although STING is best known for its role in immune responses to cytoplasmic DNA sensed by cGAS[21], 
and microglia utilize the CGAS-STING pathway to orchestrate the antiviral response in the brain[22], the molecular mechanisms underlying cGAS-STING pathway contributions to depression-like behaviors remains unknown. Here, an intriguing suggestion that arises from the present study is that activation of the STING-TBK1-IRF3 pathway might be served as a potential mechanism to boost microglia phagocytosis through promoting the production of IFN- $\beta$ in chronic restraint stress mice, consequently alleviating neuroinflammation and ameliorating depression-like behavior.

Of particular interest is that human microglia constitutively express cGAS and its critical downstream adaptor STING[23]. In a Parkinson's disease mouse model, STING-mediated inflammation has been implicated in promoting microglia activation and neuronal death[19]. Microglia are activated via the cGAS-STING pathway to induce the production of interferons to launch antiviral defense pathways[24]. Moreover, in the multiple sclerosis model[15], the antiviral drug Ganciclovir inhibits inflammation and leads to protection through activation of the STING pathway. It is interesting to note that excessive engagement of the cGAS-STING pathway in the brain can lead to neuroinflammation and neurodegeneration. It is clearly indicated that the number of microglia in certain stress-sensitive brain regions is increased during chronic stress[4]. This result was also confirmed in our study, indicating that microglia are increased in number and more activated towards M1 phenotypes in prefrontal cortex and hippocampus of RST mice. The expressions of M1-polarized microglia markers as well as the production of pro-inflammatory cytokines were increased in RST mice. Interestingly, in BV2 microglia, we observed that M1-polarized microglia have the lower level of STING expression. Thus, we wondered that whether activation of the STING pathway could ameliorate chronic restraint stress-induced depressive symptoms and relieve neuroinflammation.

CDG, CDA, 3'3-cGAMP, and 2'3-cGAMP are the native agonists of STING[25, 26]. 2'3-cGAMP, as a highaffinity ligand of STING, is a non-classical cyclic dinucleotide that activates STING and triggers potent immune responses $[14,27]$. The cGAS-STING pathway is a dual-edged sword, which may be activated or inhibited to arrive at the desired outcome. The activation of STING plays a pivotal role in mediating innate immune responses and in removing multiple pathogens including both viruses and bacteria[28, 29]. In contrast, in chronic neurodegenerative states such as the prion disease, activation of the cGAS-STING signaling pathway ameliorated inflammation and disease progression[30]. Supporting these data, other studies showed that STING activation worsens acute pancreatitis severity in experimental models[31], but plays a protective role in chronic pancreatitis models[32]. Although the detrimental and protective roles of STING in regulating immune response have been studied, the precise role of STING signaling pathway in the chronic stress model has not been clarified. We first observed that STING activation reduced levels of brain pro-inflammatory cytokines or chemokines and ameliorated depression-like behaviors in RST mice.

In the brain, STING, which elicits the interferon response, is expressed predominantly in microglia[33]. Microglia plays a major role in the non-autonomous clearance of protein aggregates in the central nervous system[34]. Previous study reported macrophage proteins that are affected by STING and demonstrated the relationship between STING and phagocytosis[35]. In agreement with this finding, we first found that microglia phagocytosis is affected by STING activation in RST mice. A question arising 
from our work relates to how cGAMP improved microglia phagocytosis in the procession of chronic stress. One possibility is that higher levels of IFN- $\beta$ caused by activation of STING signaling pathway may have contributed to the enhanced phagocytosis and anti-depressive effect observed in the present study. It should be noted that IFN- $\beta$ is also central mediator of CNS inflammation during autoimmunity, where depending on the disease, they can be either protective or detrimental[36]. Previously, Toll/interleukin-1 receptor domain-containing adapter inducing IFN- $\beta$ (TRIF) deficient microglia exhibited an increased threshold for activation of interferon-regulated genes, suggesting that IFN- $\beta$ may enhance phagocytic activity[37]. In experimental autoimmune encephalomyelitis, IFN- $\beta$ producing microglia mediated an enhanced removal of myelin debris compared to IFN- $\beta$ non-producing microglia[38]. Indeed, we observed that STING-activated microglia exhibit a superior phagocytic capacity, and IFN- $\beta$ might guide positioning of microglia in the inflamed central nervous system during chronic stress. In vitro, we observed the similar results that cGAMP-treated microglia displayed an increase in phagocytotic rates with LPS stimulation. However, without LPS stimulation, STING activation does not affect microglia phagocytosis, although the release of IFN- $\beta$ is significantly increased. In support of this, other study confirmed that treatment of macrophages with exogenous IFN- $\beta$ alone is not sufficient to induce the production of pro-inflammatory cytokines, but retreatment with IFN- $\beta$ could lead to an increase in the anti-inflammatory cytokine and a decrease in pro-inflammatory cytokine expression in response to LPS stimulation[39]. On the other hand, a slight decrease was observed in open field activity in the IFN- $\beta$-treated mice, and this amelioration of sickness was associated with trivial IL-6 production[40]. Hence, we concluded that microglia phagocytosis was enhanced via cGAMP-STING-dependent IFN- $\beta$ signaling in chronic restraint stress model, which drives behavioral changes. Besides microglia phagocytosis, neurogenesis impairments[41], dysregulation of tryptophan-kynurenine metabolism[40] and dysfunction of the HPA axis[42] were also contributed to the depression-like behaviors. More detail of mechanism should be given in future study.

In addition, STING pathway activation is linked to multiple inhibitory feedback loops, such as inflammasome activation, autophagy induction, and autocrine IFN signaling. A previous study confirmed that chronic activation of the STING pathway is associated with reduced mTORC1 signaling in metabolically relevant tissues[43]. The STING pathway is also involved in interacting with the autophagy or mitophagy machinery in innate immune responses[44-46]. Moreover, another study revealed that STING knockout could attenuate cardiac injury by inhibiting NLRP3 inflammasome mediated inflammation, apoptosis and pyroptosis[13]. Further investigations will be needed to elucidate the molecular details, as well as the consequences of the interaction between STING and other signaling pathways in different diseases.

\section{Conclusion}

In summary, our study is the first to elucidate the mechanism by which cGAMP treatment boosts microglia phagocytosis through activating IFN- $\beta$ response in a STING-dependent manner. Furthermore, this current study demonstrated that the STING-TBK1-IRF3 pathway as a key player in mediating chronic stress-induced low-grade neuroinflammation has indicated an exciting new direction to elucidate the mechanism underlying psychosocial diseases.

Page 12/25 


\section{Abbreviations}

CCL2

C-C motif chemokine ligand 2

CDA

cyclic di-adenosine

CDG

Cyclic di-GMP

cGAMP

cyclic-GMP-AMP

cGAS

cyclic dinucleotide synthetase

CNS

central nervous system

CON

control

CXCL10

C-X-C motif chemokine ligand 10

HSV-1

herpes simplex virus-1

IFN- $\beta$

interferon- $\beta$

IL-10

interleukin-10

IL-1 $\beta$

interleukin-1 $\beta$

IL-6

interleukin-6

IRF3

transcription factor interferon regulatory factor 3

LPS

lipopolysaccharide

mTORC1

mammalian target of rapamycin complex 1

NLRP3

Nod-like receptor protein 3

qRT-PCR

quantitative real-time PCR

ROI

polygonal region of interest. 
RST

restraint stress

SiRNA

small interfering RNA

STING

Stimulator of interferon gene

TBK1

TANK-binding kinase 1

TLR

toll-like receptor

TNF-a

tumor necrosis factor- $a$

TRIF

Toll/interleukin-1 receptor domain-containing adapter inducing IFN- $\beta$

\section{Declarations}

\section{Ethics approval and consent to participate}

This study was approved by the ethics committee at the First Affiliated Hospital, Xi'an Jiaotong University.

\section{Consent for publication}

Not applicable.

\section{Availability of data and materials}

All data generated or analyzed during this study are included in this published article.

\section{Competing interests}

The authors declared that they had no conflicts of interest with respect to their research, authorship or the publication of this article.

\section{Funding}

This work was supported by funds from the National Natural Science Foundation of China (Grant Nos. 81901237, 81774113, and 81974540), Beijing, China.

\section{Authors' contributions}

QZ, YZ and QW conceived the study; QZ and ST performed the study; YZ, ST, JS, MY, KP, MW and HG performed the analyses; QZ and YZ wrote the paper. All authors commented on the paper. 


\section{Acknowledgements}

We would also like to acknowledge the support of Yan Li and Ying Zhang on this project.

\section{References}

1. Quinlan, E.B., et al., Psychosocial Stress and Brain Function in Adolescent Psychopathology. Am J Psychiatry, 2017. 174(8): p. 785-794.

2. Salam, A.P., A. Borsini, and P.A. Zunszain, Trained innate immunity: a salient factor in the pathogenesis of neuroimmune psychiatric disorders. Mol Psychiatry, 2018. 23(2): p. 170-176.

3. Zhu, Y., et al., Neuroinflammation caused by mental stress: the effect of chronic restraint stress and acute repeated social defeat stress in mice. Neurol Res, 2019. 41(8): p. 762-769.

4. Tynan, R.J., et al., Chronic stress alters the density and morphology of microglia in a subset of stressresponsive brain regions. Brain Behav Immun, 2010. 24(7): p. 1058-68.

5. Delpech, J.C., et al., Early life stress perturbs the maturation of microglia in the developing hippocampus. Brain Behav Immun, 2016. 57: p. 79-93.

6. Nie, X., et al., The Innate Immune Receptors TLR2/4 Mediate Repeated Social Defeat Stress-Induced Social Avoidance through Prefrontal Microglial Activation. Neuron, 2018. 99(3): p. 464-+.

7. Yue, N., et al., Activation of P2X7 receptor and NLRP3 inflammasome assembly in hippocampal glial cells mediates chronic stress-induced depressive-like behaviors. J Neuroinflammation, 2017. 14(1): p. 102.

8. Ishikawa, H. and G.N. Barber, STING is an endoplasmic reticulum adaptor that facilitates innate immune signalling (vol 455, pg 674, 2008). Nature, 2008. 456(7219): p. 274-274.

9. Konno, H., K. Konno, and G.N. Barber, Cyclic dinucleotides trigger ULK1 (ATG1) phosphorylation of STING to prevent sustained innate immune signaling. Cell, 2013. 155(3): p. 688 - 98.

10. Hara, M.R., et al., A stress response pathway regulates DNA damage through beta2-adrenoreceptors and beta-arrestin-1. Nature, 2011. 477(7364): p. $349-53$.

11. Burdette, D.L., et al., STING is a direct innate immune sensor of cyclic di-GMP. Nature, 2011. 478(7370): p. 515-8.

12. Radoshevich, L. and O. Dussurget, Cytosolic Innate Immune Sensing and Signaling upon Infection. Frontiers in Microbiology, 2016. 7.

13. Li, N., et al., STING-IRF3 contributes to lipopolysaccharide-induced cardiac dysfunction, inflammation, apoptosis and pyroptosis by activating NLRP3. Redox Biol, 2019. 24: p. 101215. 
14. Sun, L.J., et al., Cyclic GMP-AMP Synthase Is a Cytosolic DNA Sensor That Activates the Type I Interferon Pathway. Science, 2013. 339(6121): p. 786-791.

15. Mathur, V., et al., Activation of the STING-Dependent Type I Interferon Response Reduces Microglial Reactivity and Neuroinflammation. Neuron, 2017. 96(6): p. 1290-1302 e6.

16. Xie, Y., et al., Effects of nanoparticle zinc oxide on spatial cognition and synaptic plasticity in mice with depressive-like behaviors. J Biomed Sci, 2012. 19: p. 14.

17. York, E.M., et al., 3DMorph Automatic Analysis of Microglial Morphology in Three Dimensions from Ex Vivo and In Vivo Imaging. eNeuro, 2018. 5(6).

18. Ohkuri, T., et al., STING contributes to antiglioma immunity via triggering type I IFN signals in the tumor microenvironment. Cancer Immunol Res, 2014. 2(12): p. 1199 - 208.

19. Sliter, D.A., et al., Parkin and PINK1 mitigate STING-induced inflammation. Nature, 2018. 561(7722): p. 258-262.

20. Gui, X., et al., Autophagy induction via STING trafficking is a primordial function of the cGAS pathway. Nature, 2019. 567(7747): p. 262-+.

21. Ahn, J., et al., STING manifests self DNA-dependent inflammatory disease. Proc Natl Acad Sci U S A, 2012. 109(47): p. 19386-91.

22. Reinert, L.S., et al., Sensing of HSV-1 by the cGAS-STING pathway in microglia orchestrates antiviral defence in the CNS. Nature Communications, 2016. 7.

23. Jeffries, A.M. and I. Marriott, Human microglia and astrocytes express cGAS-STING viral sensing components. Neurosci Lett, 2017. 658: p. 53-56.

24. Gulen, M.F., et al., Signalling strength determines proapoptotic functions of STING. Nat Commun, 2017. 8(1): p. 427.

25. Feng, X., et al., Bioactive modulators targeting STING adaptor in CGAS-STING pathway. Drug Discov Today, 2020. 25(1): p. 230-237.

26. Wu, J.J., et al., Agonists and inhibitors of the STING pathway: Potential agents for immunotherapy. Med Res Rev, 2020. 40(3): p. 1117-1141.

27. Cai, X., Y.H. Chiu, and Z.J. Chen, The cGAS-cGAMP-STING pathway of cytosolic DNA sensing and signaling. Mol Cell, 2014. 54(2): p. $289-96$.

28. Cohen, D., et al., Cyclic GMP-AMP signalling protects bacteria against viral infection. Nature, 2019. 574(7780): p. 691-695. 
29. Xu, Q., et al., Efficacy and mechanism of cGAMP to suppress Alzheimer's disease by elevating TREM2. Brain Behav Immun, 2019. 81: p. 495-508.

30. Nazmi, A., et al., Chronic neurodegeneration induces type I interferon synthesis via STING, shaping microglial phenotype and accelerating disease progression. Glia, 2019. 67(7): p. 1254-1276.

31. Zhao, Q., et al., STING Signaling Promotes Inflammation in Experimental Acute Pancreatitis. Gastroenterology, 2018. 154(6): p. 1822-1835 e2.

32. Zhao, Q.L., et al., STING signalling protects against chronic pancreatitis by modulating Th17 response. Gut, 2019. 68(10): p. 1827-+.

33. Tejera, D. and M.T. Heneka, United Again: STING and the Police. Neuron, 2017. 96(6): p. 1207-1208.

34. Kemeny, M.E. and M. Schedlowski, Understanding the interaction between psychosocial stress and immune-related diseases: A stepwise progression. Brain Behavior and Immunity, 2007. 21(8): p. 10091018.

35. Aryal, U.K., et al., Global Proteomic Analyses of STING-Positive and-Negative Macrophages Reveal STING and Non-STING Differentially Regulated Cellular and Molecular Pathways. Proteomics Clin Appl, 2020: p. e1900109.

36. Deczkowska, A., K. Baruch, and M. Schwartz, Type I/IIInterferon Balance in the Regulation of Brain Physiology and Pathology. Trends Immunol, 2016. 37(3): p. 181-192.

37. Hosmane, S., et al., Toll/interleukin-1 receptor domain-containing adapter inducing interferon-beta mediates microglial phagocytosis of degenerating axons. J Neurosci, 2012. 32(22): p. 7745-57.

38. Scheu, S., et al., Interferon -Mediated Protective Functions of Microglia in Central Nervous System Autoimmunity. International Journal of Molecular Sciences, 2019. 20(1).

39. Karimi, Y., et al., IFN-beta signalling regulates RAW 264.7 macrophage activation, cytokine production, and killing activity. Innate Immun, 2020. 26(3): p. 172-182.

40. Murray, C., et al., Interdependent and independent roles of type I interferons and IL-6 in innate immune, neuroinflammatory and sickness behaviour responses to systemic poly I:C. Brain Behav Immun, 2015. 48: p. $274-86$.

41. Han, Y., et al., Pioglitazone alleviates maternal sleep deprivation-induced cognitive deficits in male rat offspring by enhancing microglia-mediated neurogenesis. Brain Behav Immun, 2020. 87: p. 568-578.

42. Zhao, X.J., et al., Activation of ATP-sensitive potassium channel by iptakalim normalizes stressinduced HPA axis disorder and depressive behaviour by alleviating inflammation and oxidative stress in mouse hypothalamus. Brain Res Bull, 2017. 130: p. 146-155. 
43. Hasan, M., et al., Chronic innate immune activation of TBK1 suppresses mTORC1 activity and dysregulates cellular metabolism. Proc Natl Acad Sci U S A, 2017. 114(4): p. 746-751.

44. Watson, R.O., P.S. Manzanillo, and J.S. Cox, Extracellular M. tuberculosis DNA targets bacteria for autophagy by activating the host DNA-sensing pathway. Cell, 2012. 150(4): p. $803-15$.

45. Liang, Q.M., et al., Crosstalk between the cGAS DNA Sensor and Beclin-1 Autophagy Protein Shapes Innate Antimicrobial Immune Responses. Cell Host \& Microbe, 2014. 15(2): p. 228-238.

46. Lei, Z., et al., cGAS-mediated autophagy protects the liver from ischemia-reperfusion injury independently of STING. Am J Physiol Gastrointest Liver Physiol, 2018. 314(6): p. G655-G667.

\section{Figures}



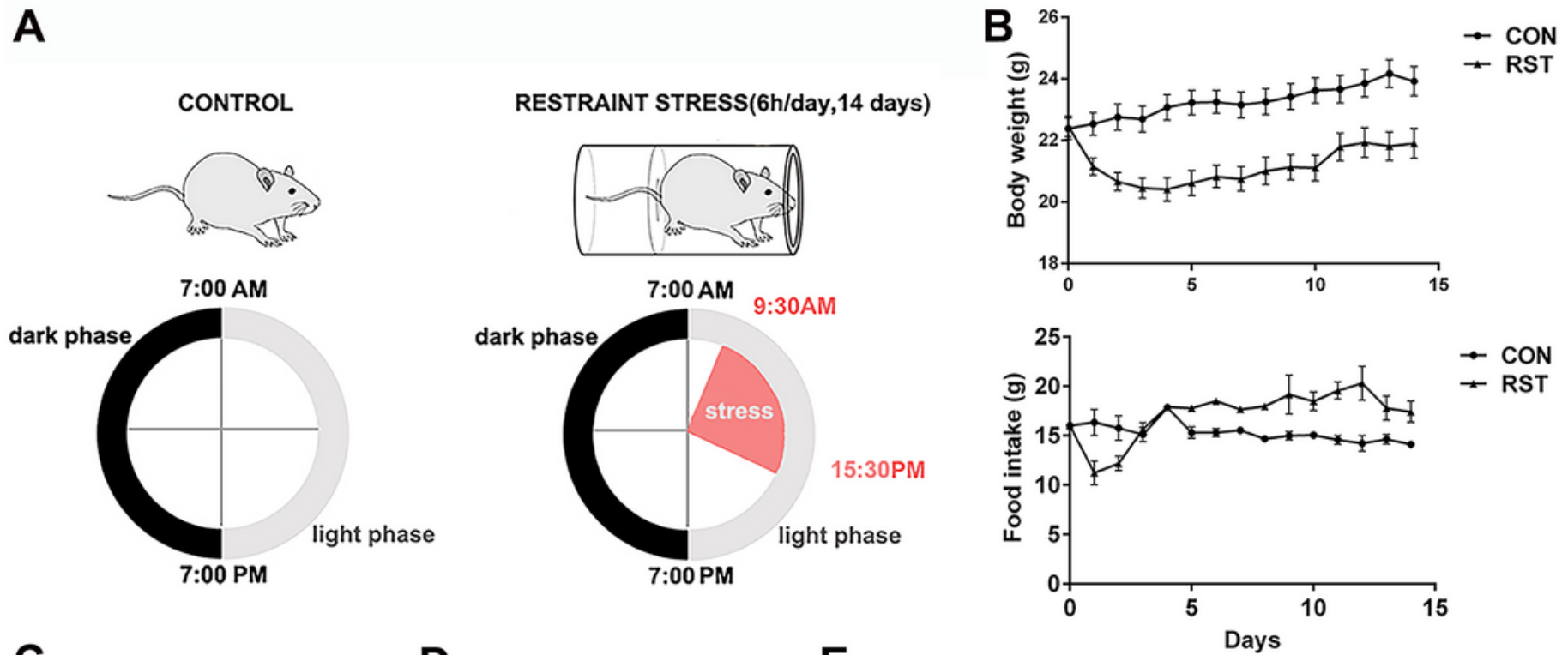

C

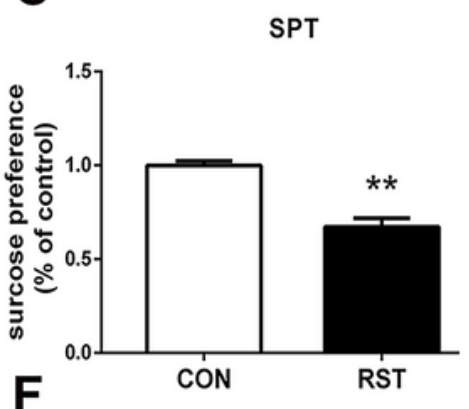

$\mathbf{F}$
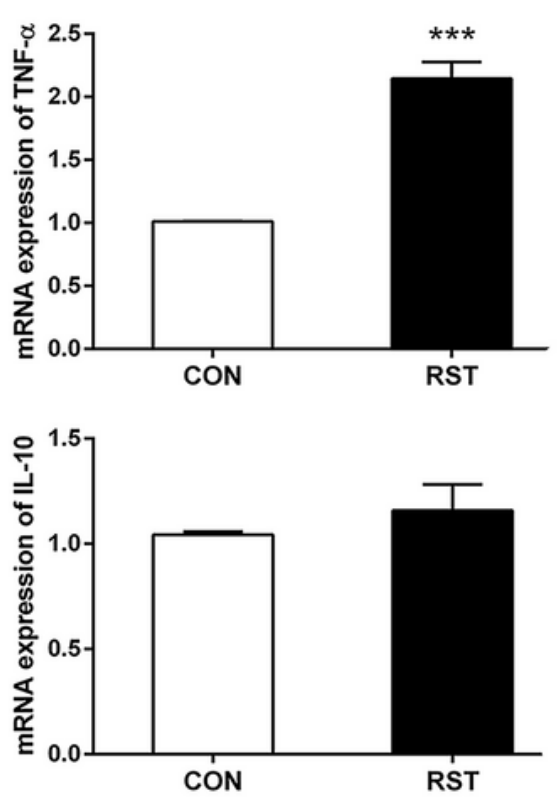

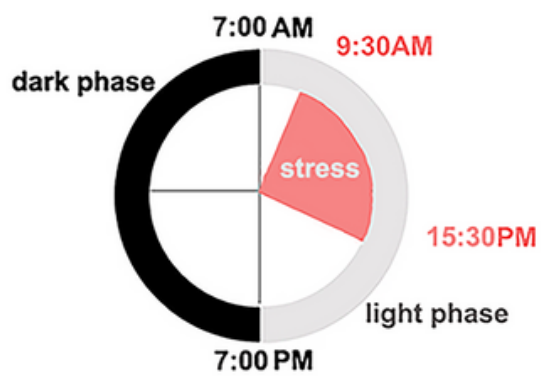

RESTRAINT STRESS(6h/day,14 days)

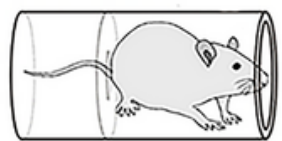

$\mathbf{E}$
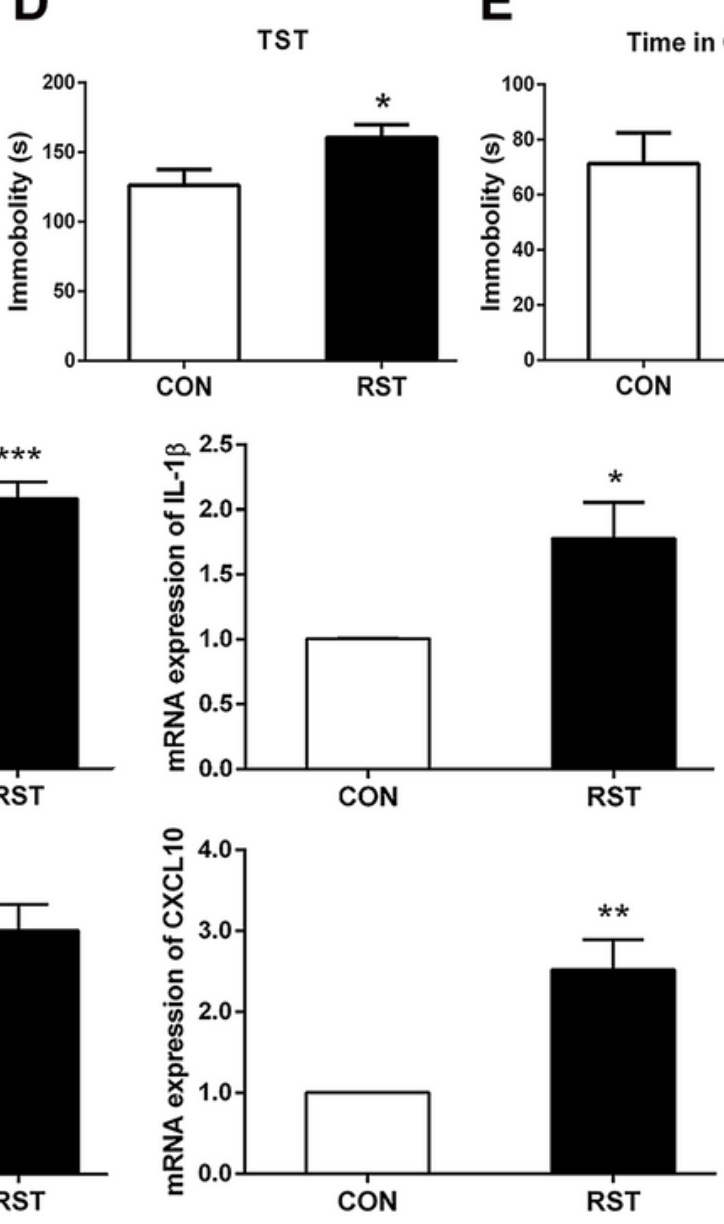

Entries in Open Arms

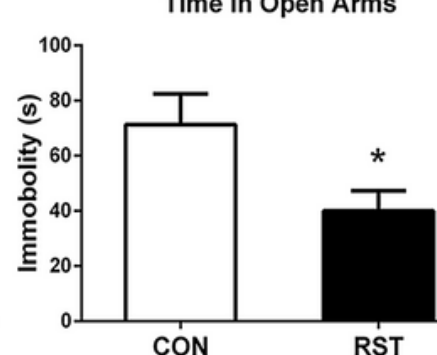

RST
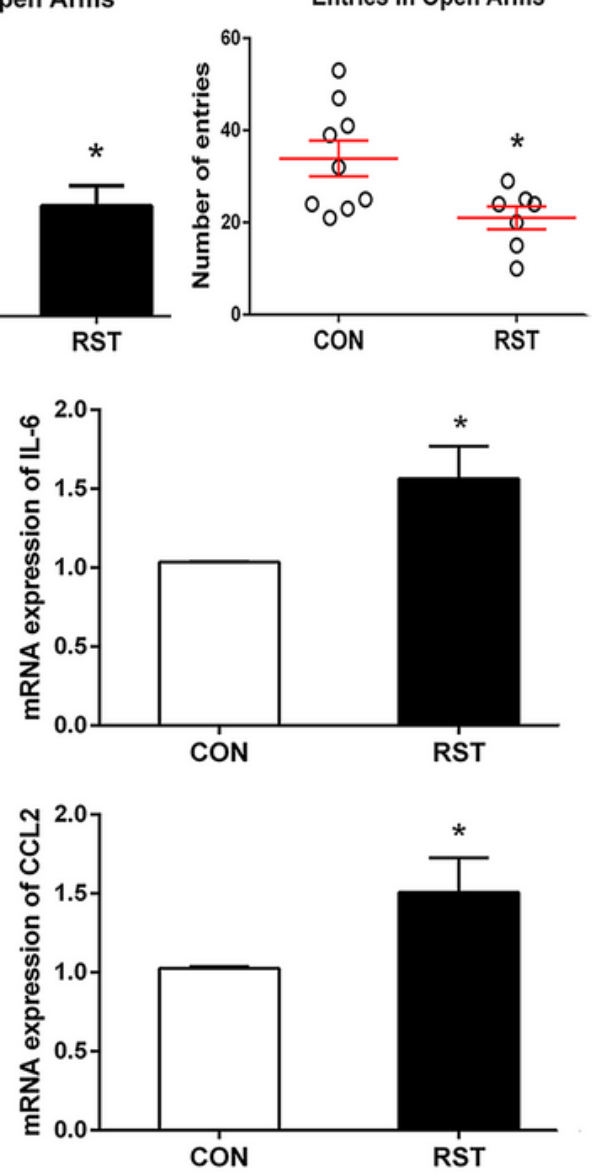

Figure 1

Chronic restraint stress induces depression-like behaviors. a Diagram of the control or chronic restraint stress protocol. b Body weight and food intake were measured daily during the 14-day according to the experimental protocol. c RST mice displayed anhedonia in a sucrose preference test. d Immobility time of RST mice was increased in the tail suspension test. e RST mice spent less time in the open arms and made fewer entries into the open arm in the elevated plus maze test. $\mathrm{f}$ Brain pro-inflammatory cytokines 
and chemokines were increased in RST mice. mRNA expression levels of TNF-a, IL-1 $\beta$, IL-6, IL-10, CXCL10 and CCL2 in brain tissues were examined using real-time PCR. Data are presented as the mean \pm SEM. *P $<0.05, * * \mathrm{P}<0.01$ and $* * * \mathrm{P}<0.001$ between CON vs. RST in t-tests ( $\mathrm{N}=7-10$ mice per group).

A

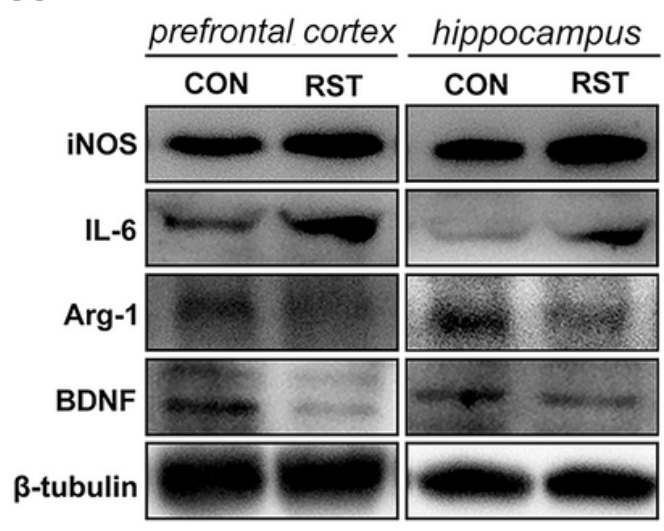

$\mathbf{F}$

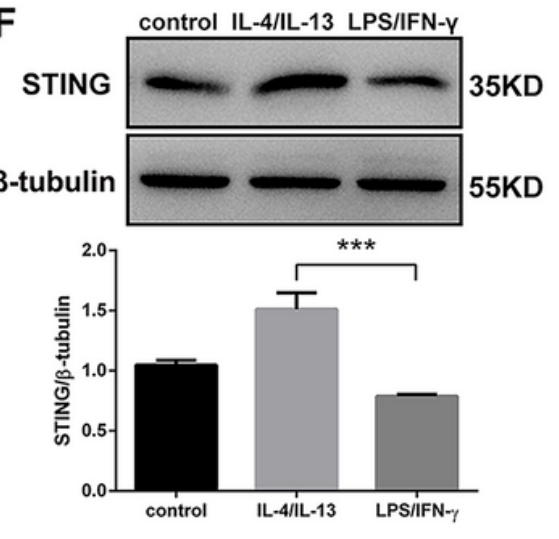

B
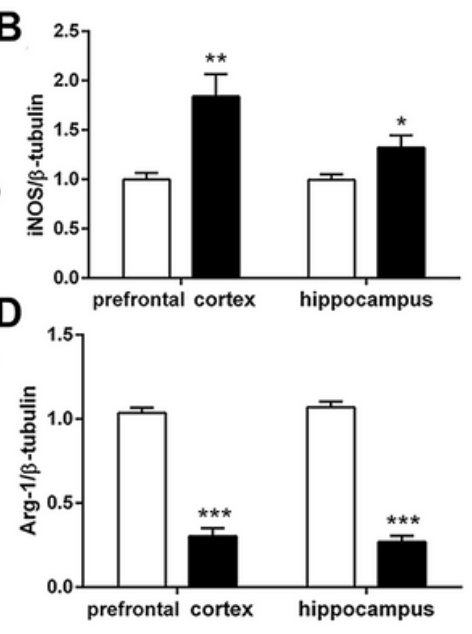

G
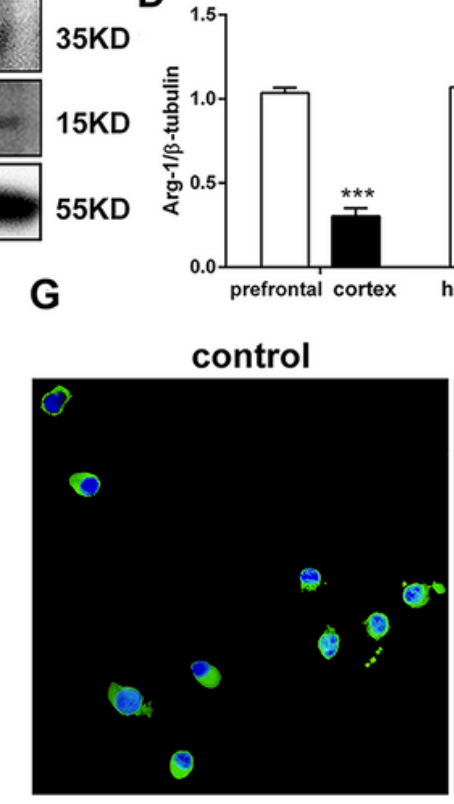

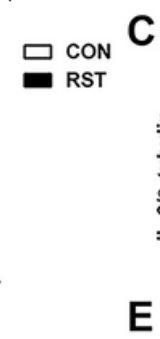

E
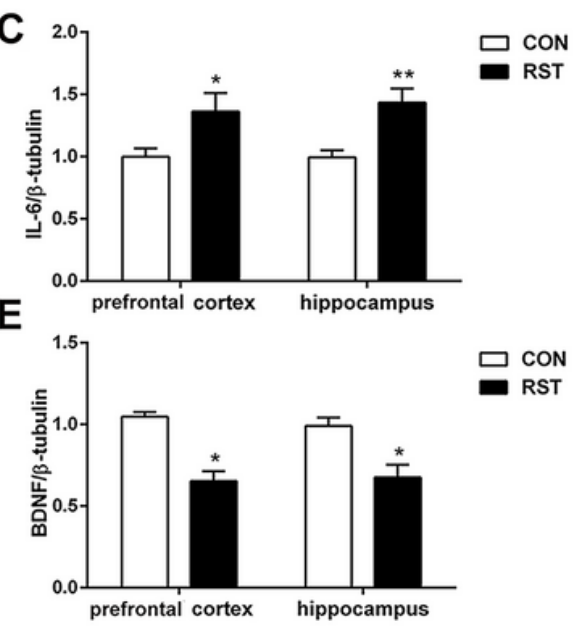

IL-4/IL-13

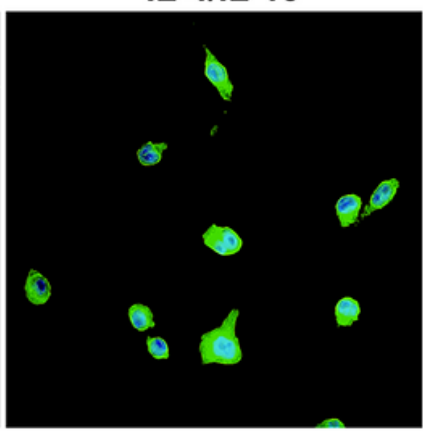

LPS/IFN-Y

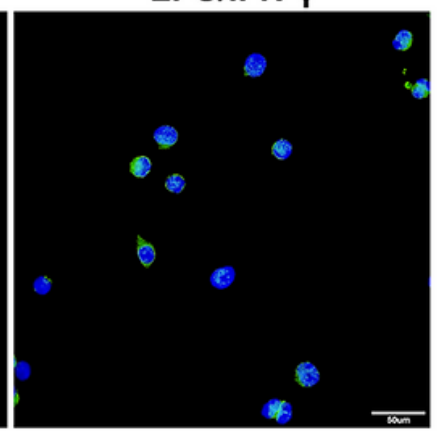

Figure 2

In RST mice, microglia were activated towards to M1 phenotype in the prefrontal cortex and hippocampus. a Photomicrographs show protein levels of induced nitric-oxide synthase (iNOS), interleukin-6 (IL-6), arginase-1 (Arg-1), and brain-derived neurotrophic factor (BDNF). $\beta$-tubulin was used as a loading control. b Protein expression of iNOS in CON and RST mice. c Protein expression of IL-6 in CON and RST mice. d Protein expression of Arg-1 in CON and RST mice. e Protein expression of BDNF in CON and RST mice. f Protein expression of STING in resting microglia, IL-4/IL-13-induced M2 phenotype microglia and LPS/IFN-y-induced M1 phenotype microglia, which are represented by green fluorescence in $\mathrm{g}$. Data are presented as the mean \pm SEM. ${ }^{\star} \mathrm{P}<0.05,{ }^{\star \star} \mathrm{P}<0.01$ and ${ }^{\star \star \star *} \mathrm{P}<0.001(\mathrm{~N}=6$ mice per group) 
A

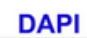

Iba1

STING

overlay

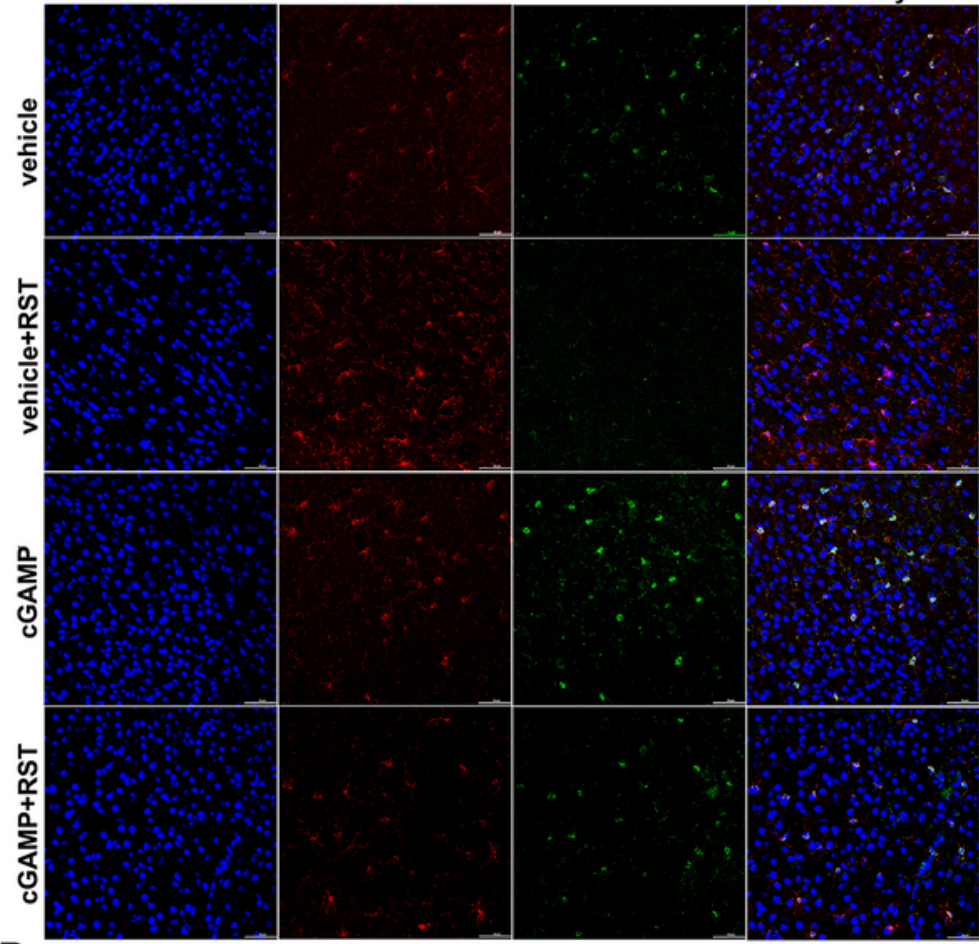

B

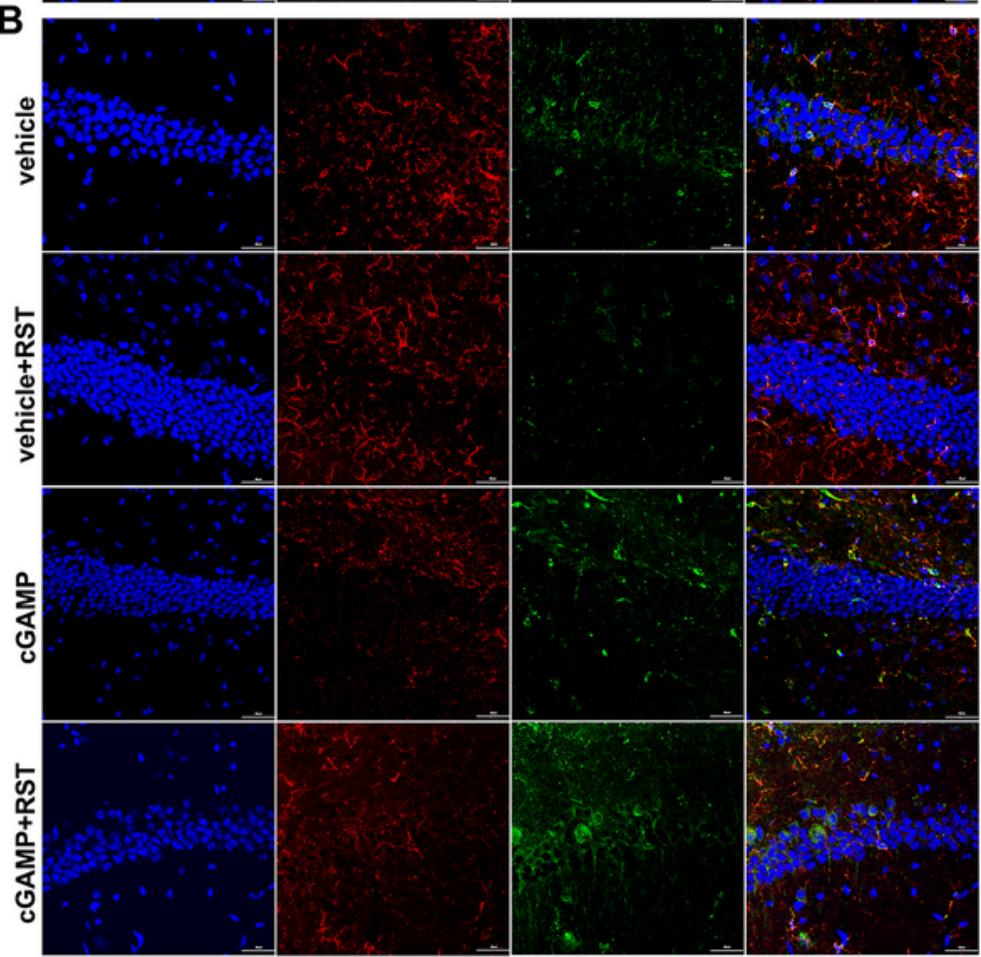

C

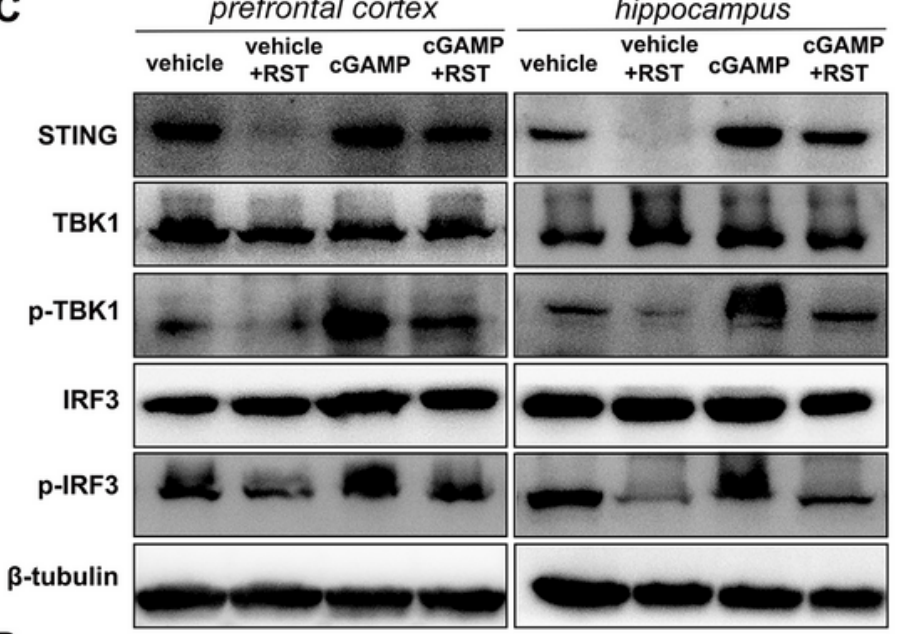

D

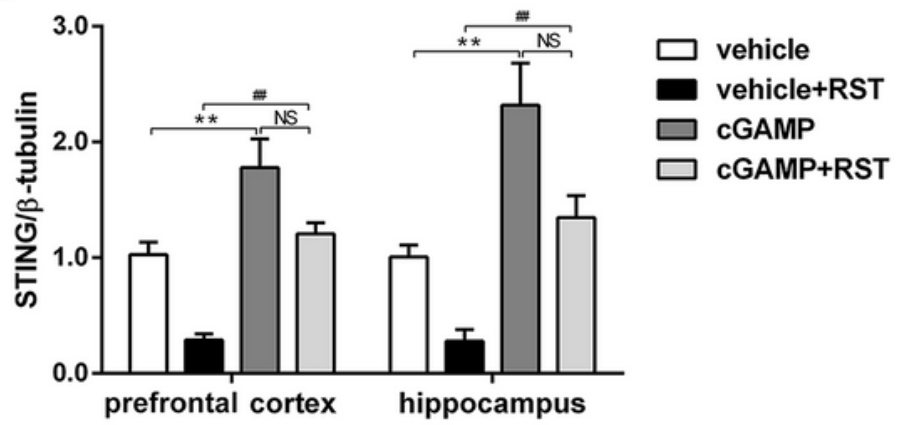

E

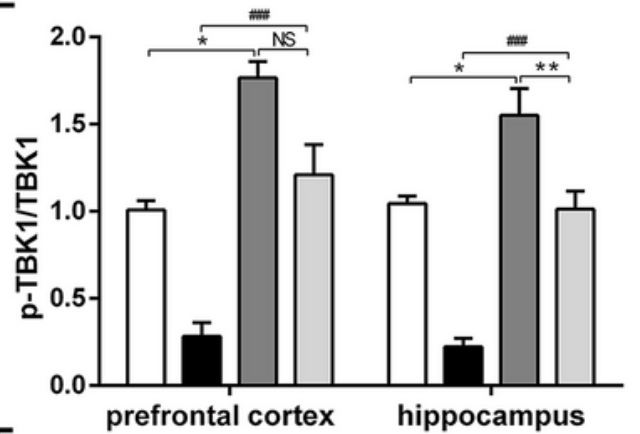

F

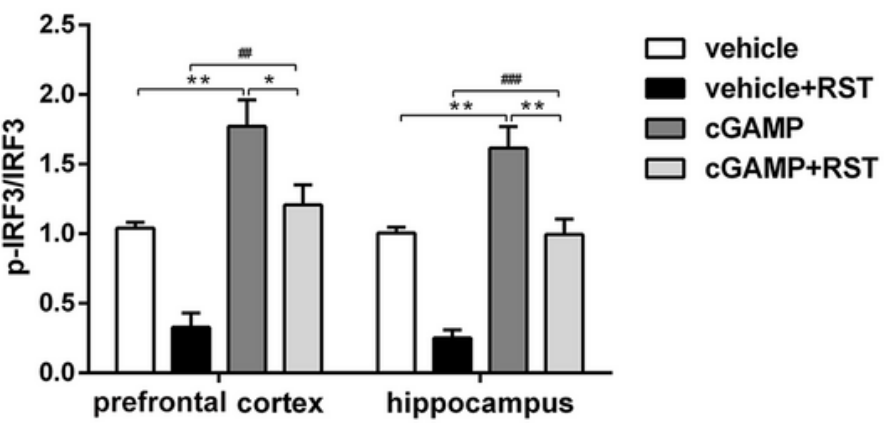

Figure 3

Suppression of the STING-TBK1-IRF3 signaling pathway in RST mice was reversed after delivery of 2'3cGAMP into the brain. a Immunofluorescent staining for lba-1 (red) and STING (green) in the prefrontal cortex of vehicle, vehicle+RST, cGAMP, and cGAMP+RST mice. b Immunofluorescent staining for Iba-1 (red) and STING (green) in the hippocampus of vehicle, vehicle+RST, cGAMP, cGAMP+RST mice. The scale bar denotes $50 \mu \mathrm{m}$. c Representative western blot analyses of protein expression levels of STING, 
TBK1, and IRF3, which were measured in the prefrontal cortex and hippocampus of vehicle, vehicle+RST, cGAMP, and cGAMP+RST mice. d Protein level of STING was significantly increased in cGAMP mice, whereas there was no change in the cGAMP+RST group compared with the vehicle group. e Increased phosphorylation of TBK1 normalized to their total protein expression was observed in cGAMP mice. There was no significant difference between the vehicle and cGAMP+RST groups. $f$ Meanwhile, increased phosphorylation of IRF3 normalized to their total protein expression was observed in cGAMP mice. $\beta$ tubulin was used to normalize the total expression of proteins. Data are presented as the mean \pm SEM. $* P$ $<0.05$, $* * P<0.01, \# P<0.01$ and \#\#\#P<0.001 ( $N=6-8$ mice per group).

\section{A}
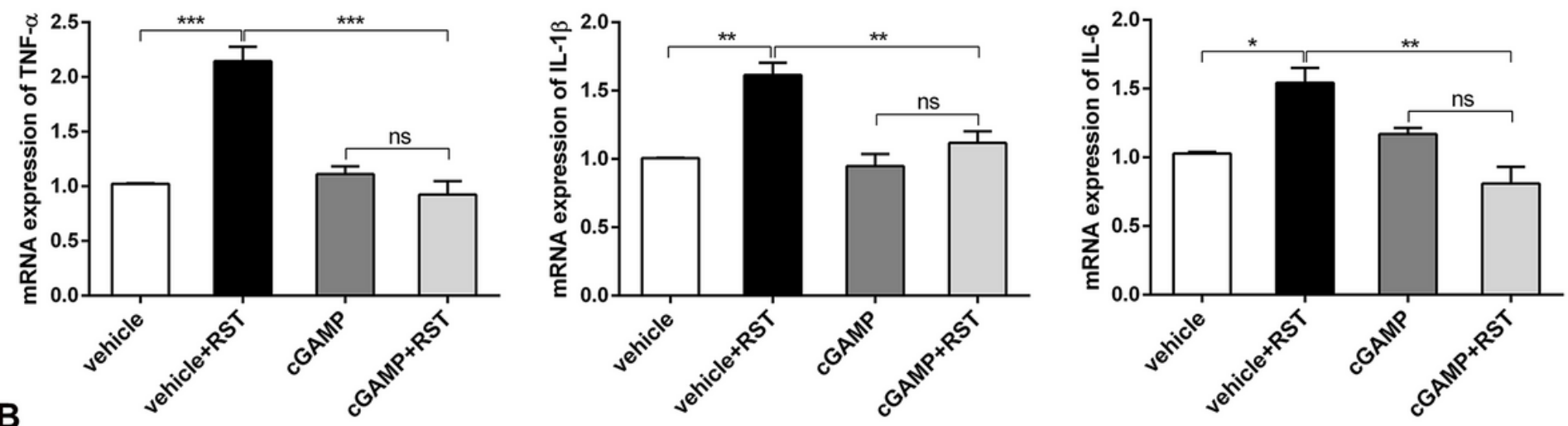

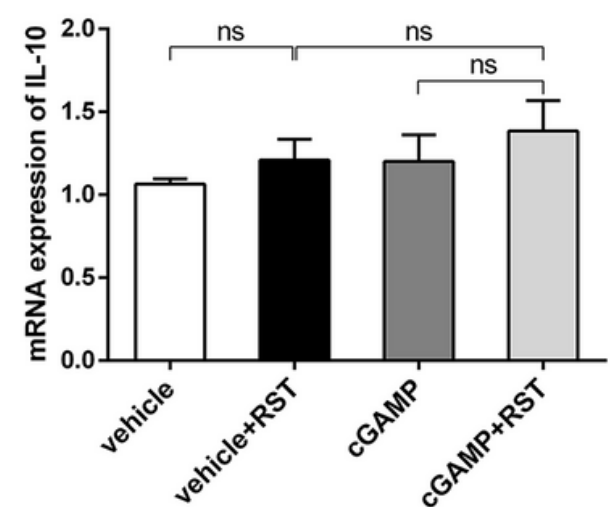

C

SPT
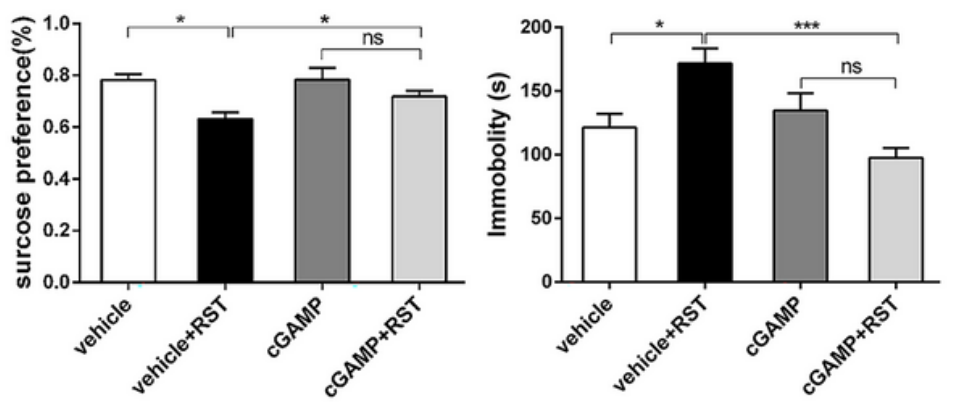

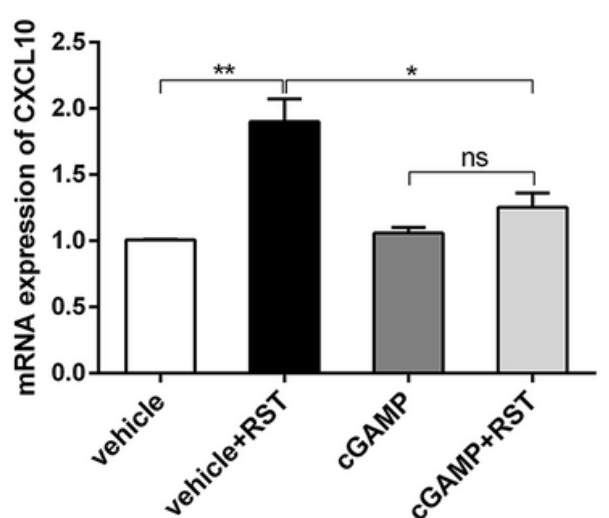

E

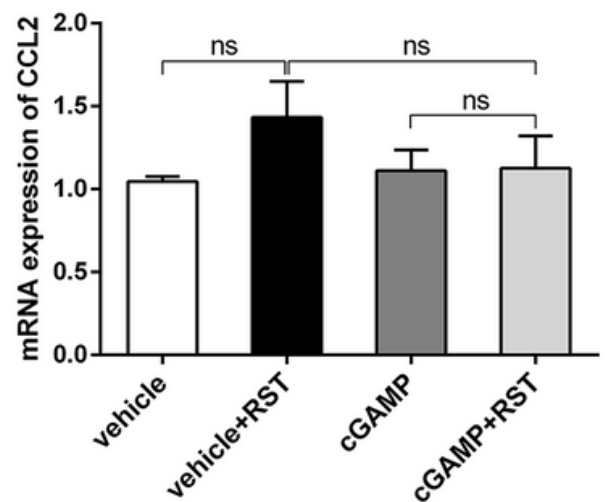

Entries in Open Arms

Time in Open Arms
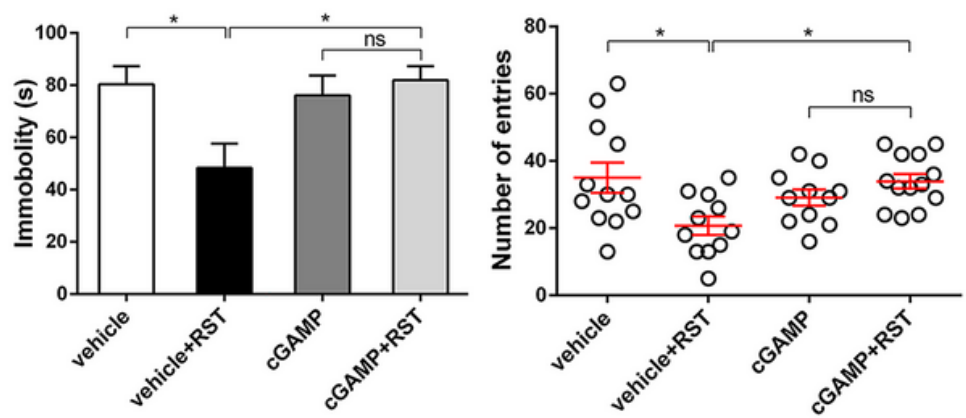

Figure 4

Effects of cGAMP on the brain inflammatory profiles and depression-like behaviors in RST mice. a mRNA expression levels of TNF- $a, \mathrm{IL}-1 \beta, \mathrm{IL}-6$ in the brains of vehicle, vehicle+RST, cGAMP, and cGAMP+RST mice. b mRNA expression of IL-10, CXCL10 and CCL2 in vehicle, vehicle+RST, cGAMP, and cGAMP+RST 
mice. c The sucrose preference ratio of vehicle, vehicle+RST, cGAMP, and cGAMP+RST mice. $d$ The immobility time of restraint stress mice treated with cGAMP was significantly reduced in the tail suspension test. e The immobility time in open arms was increased in cGAMP+RST group compared with that in vehicle+RST group, and the total number of open arm entries in cGAMP+RST mice was also increased. Data are presented as the mean \pm SEM. ${ }^{*} \mathrm{P}<0.05$ and ${ }^{*} \mathrm{P}<0.01(\mathrm{~N}=10-12$ mice per group).

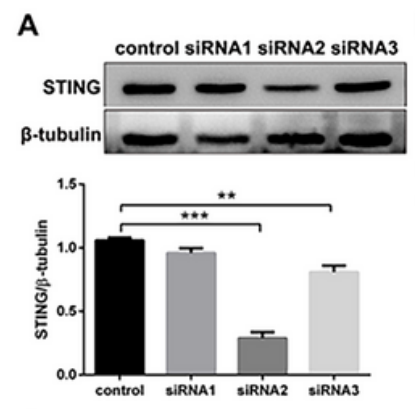

c
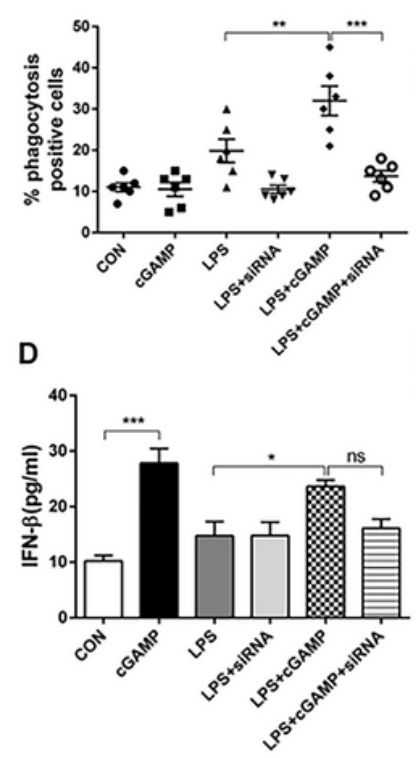

G

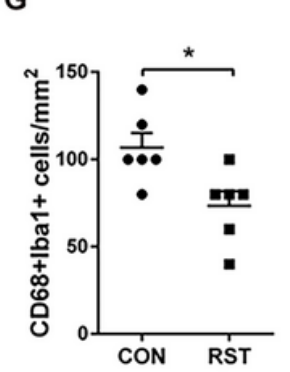

B
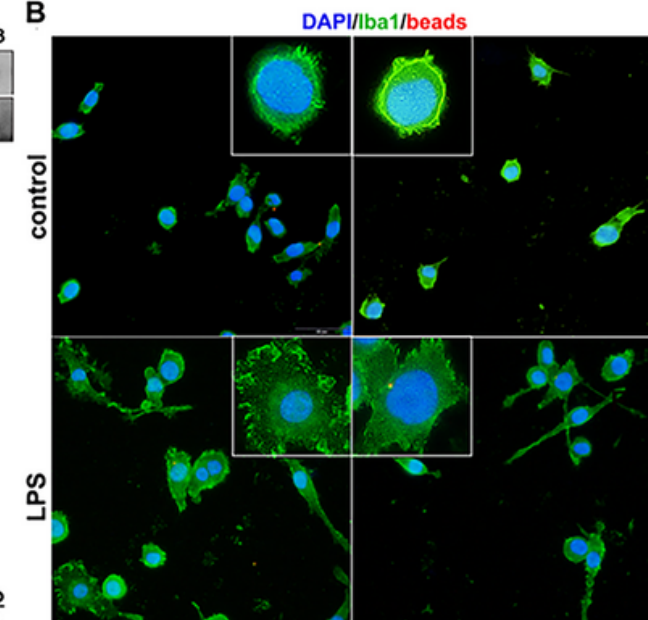

E

DAPI

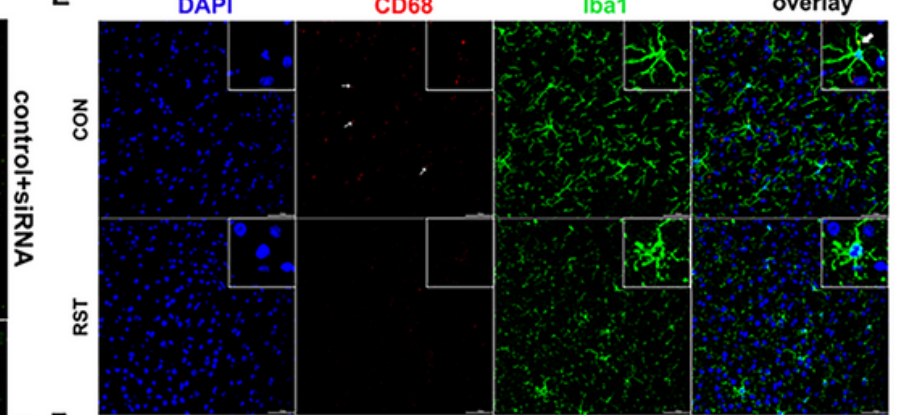

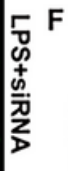
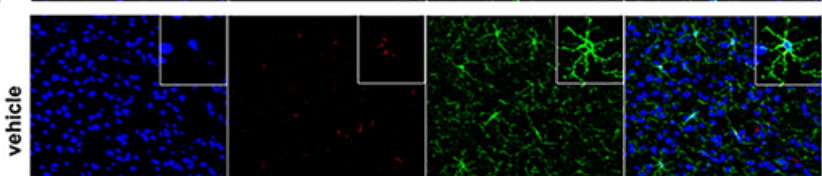

$\mathbf{H}$

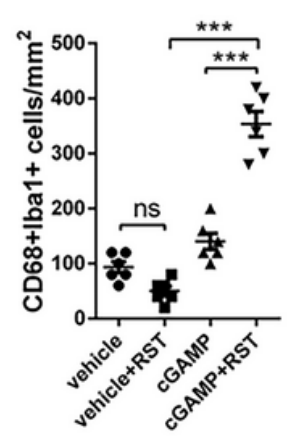

1

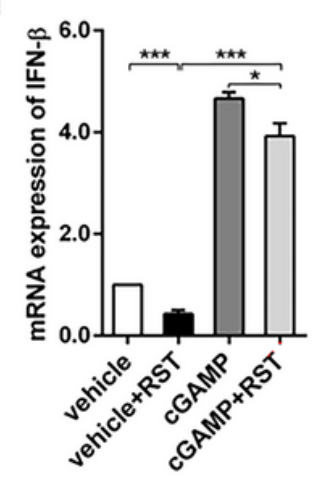

$\mathbf{J}$

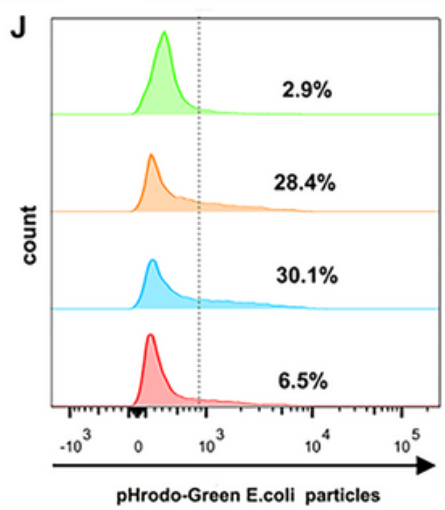

LPS

LPS+rIFN

= LPS+CGAMP

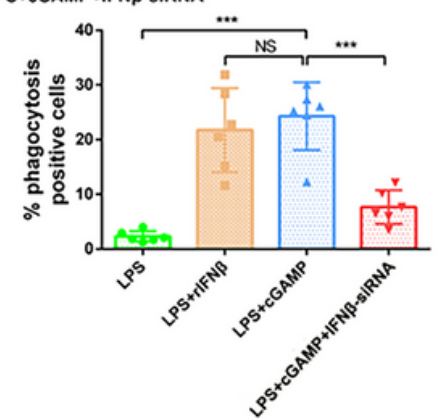

\section{Figure 5}

GAMP treatment promoted microglia phagocytosis by inducing the production of IFN- $\beta$. a Western blotting showed the effect of STING-small interfering RNA (siRNA) on the expression of STING in BV2 microglia. b Representative fluorescent images showing staining of cell nuclei (DAPI, blue), latex beads (red) and microglia (Iba1, green) $2 \mathrm{~h}$ after incubation of amino-modified polystyrene latex beads. Scale 
bars represent $50 \mu \mathrm{m}$. c Statistical analysis of phagocytosis positive cells in each group. $d$ The level of IFN- $\beta$ in BV2 cell supernatant of each group. e Representative micrographs show microglia (lba1 staining, green), CD68 (red) and cell nuclei (DAPI, blue) in the prefrontal cortex of CON and RST mice. The scale bar denotes $50 \mu \mathrm{m}$. f Representative micrographs show microglia (lba1 staining, green), CD68 (red) and cell nuclei (DAPI, blue) in the prefrontal cortex of vehicle, vehicle+RST, cGAMP, and cGAMP+RST mice. The scale bar denotes $50 \mu \mathrm{m}$. g Statistical analysis of CD68+lba1+ cells in CON and RST mice. $\mathrm{h}$ Statistical analysis of CD68+lba1+ cells in vehicle, vehicle+RST, cGAMP, and cGAMP+RST mice. i mRNA expression of IFN- $\beta$ in the brains of vehicle, vehicle+RST, cGAMP, and cGAMP+RST mice. j LPS (green), LPS+recombinant-IFN $\beta$ (orange), LPS+cGAMP (blue)- and LPS+cGAMP+IFNß-siRNA (red)-dependent phagocytosis in BV2 microglia. Bar graph quantifying percentage of E. coli bioparticles with FITC fluorescent intensity. ${ }^{*} \mathrm{P}<0.05$, ${ }^{*} \mathrm{P}<0.01$ and ${ }^{*} * \mathrm{P}<0.001$. ( $\mathrm{N}=6-8$ mice per group).

A
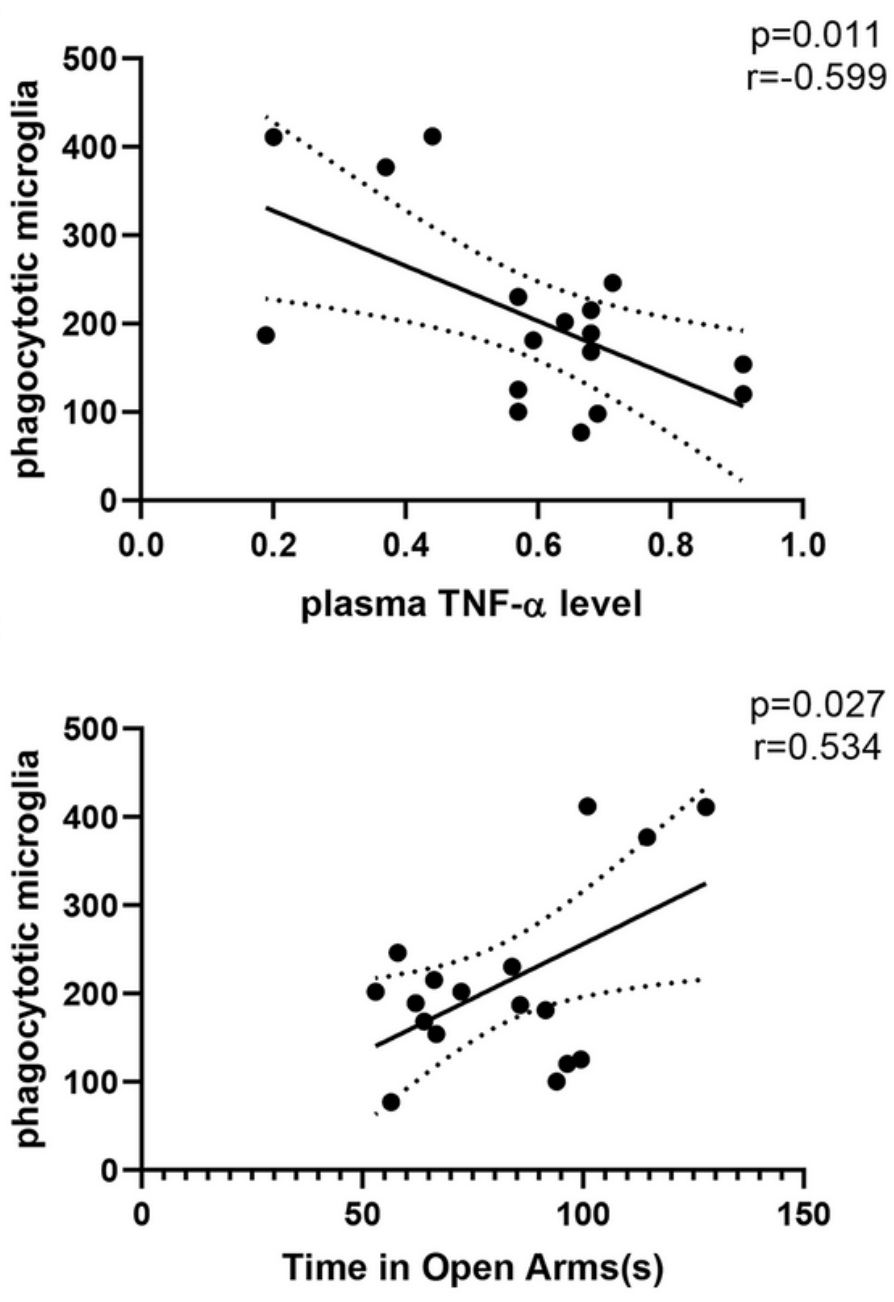

B
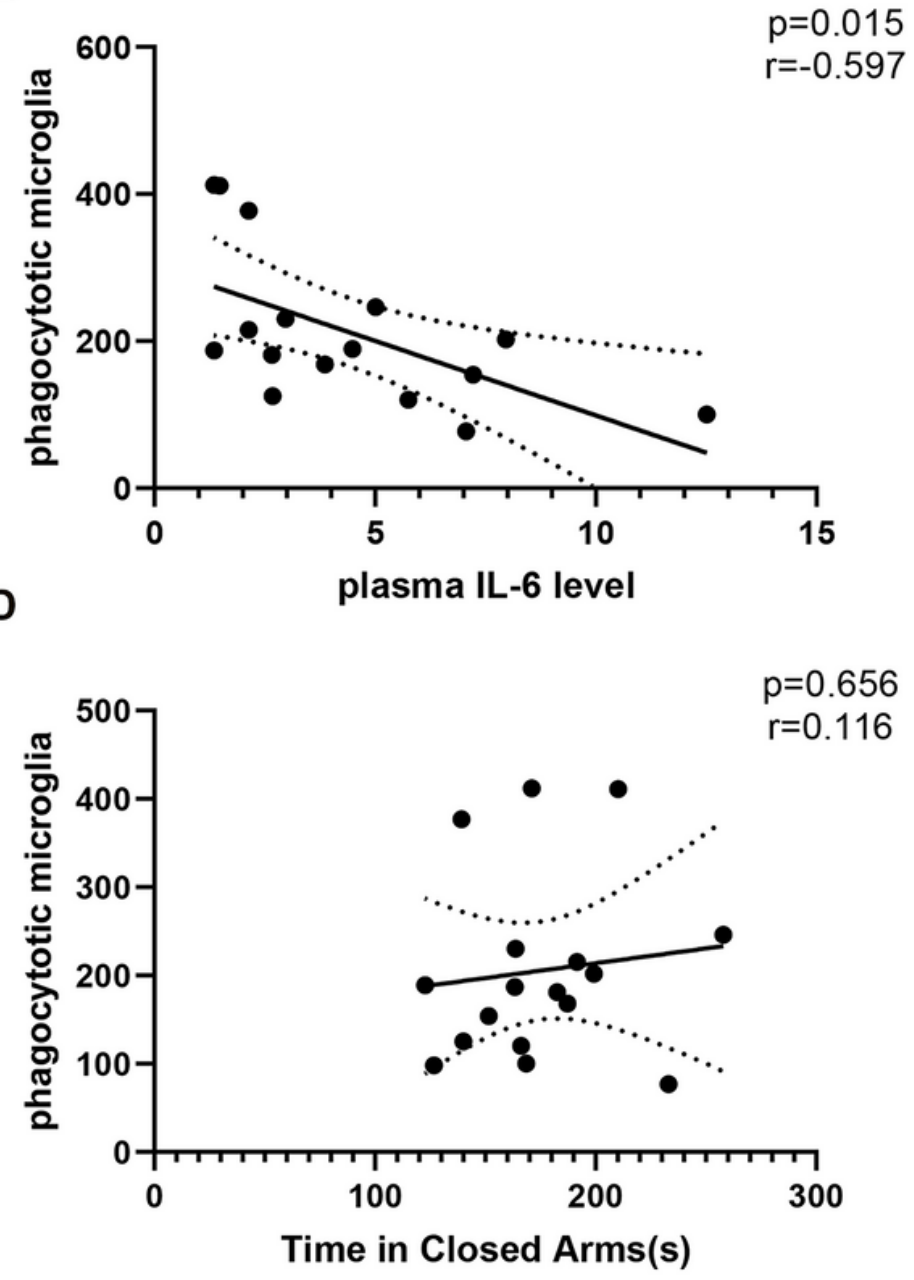

Figure 6

Pearson correlations support that STING activation alleviates chronic restraint stress-induced neuroinflammation and ameliorates depression-like behavior through boosting microglia phagocytosis. a Plasma TNF-a level and b plasma IL-6 level were significantly inversely correlated with phagocytic microglia cell counts. c Times in open arms was significantly positively correlated with phagocytic 
microglia cell counts. $d$ There was no significant correlation between phagocytic microglia cell counts and times in closed arms.

\section{Supplementary Files}

This is a list of supplementary files associated with this preprint. Click to download.

- supplementaryfigure1.tif

- supplementaryfigure2.tif

- supplementaryfigure3.tif

- supplementarymaterialsandmethods.doc 